\title{
Bovine Meniscus Middle Zone Tissue: Measurement of Collagen Fibril Behavior During Compression
}

This article was published in the following Dove Press journal:

International Journal of Nanomedicine

\author{
Katie H Sizeland (D) \\ Hannah C Wells ${ }^{2}$ \\ Nigel M Kirby ${ }^{3}$ \\ Adrian Hawley ${ }^{3}$ \\ Stephen T Mudie $\mathbb{D}^{3}$ \\ Tim M Ryan ${ }^{3}$ \\ Richard G Haverkamp $\mathbb{D}^{2}$ \\ 'Human Health, ANSTO, Lucas Heights, \\ Sydney, NSW 2234, Australia; ${ }^{2}$ School of \\ Food and Advanced Technology, Massey \\ University, Palmerston North 4472, New \\ Zealand; ${ }^{3}$ SAXS/WAXS Beamline, \\ Australian Synchrotron, ANSTO, \\ Clayton, Melbourne, VIC 3168, Australia
}

Correspondence: Richard G Haverkamp School of Food and Advanced Technology, Massey University, Private Bag II I2,

Palmerston North 4472, New Zealand

Tel +64 63569099

Email r.haverkamp@massey.ac.nz
Background: Type I collagen is the major component of the extracellular matrix of the knee's meniscus and plays a central role in that joint's biomechanical properties. Repair and reconstruction of tissue damage often requires a scaffold to assist the body to rebuild. The middle zone of bovine meniscus is a material that may be useful for the preparation of extracellular matrix scaffolds.

Methods: Here, synchrotron-based small-angle X-ray scattering (SAXS) patterns of bovine meniscus were collected during unconfined compression. Collagen fibril orientation, D-spacing, compression distance and force were measured.

Results: The collagen fibrils in middle zone meniscal fibrocartilage become more highly oriented perpendicular to the direction of compression. The D-spacing also increases, from 65.0 to $66.3 \mathrm{~nm}$ with compression up to $0.43 \mathrm{MPa}$, representing a $1.8 \%$ elongation of collagen fibrils perpendicular to the compression.

Conclusion: The elasticity of the collagen fibrils under tension along their length when the meniscus is compressed, therefore, contributes to the overall elastic response of the meniscus only under loads that exceed those likely to be experienced physiologically.

Keywords: collagen, meniscus, compression, SAXS, scaffold

\section{Introduction}

Major repair and reconstruction of tissue often requires a scaffold to assist the body to rebuild. A wide variety of materials have been developed or investigated for this purpose, often tailored to a particular set of applications. ${ }^{1,2}$ These may be derived from decellularised natural tissues, produced from reconstructed collagen or manufactured from other materials. ${ }^{3-6}$ Decellularised tissue for scaffolds brings with them particular arrangements of extracellular matrix (ECM) with some more suitable for particular applications than others. ${ }^{7-10}$ A detailed understanding of tissue structures as suitable candidates for the production of ECM scaffolds by decellularisation is desirable to add to the collection of materials available. ${ }^{11}$

The middle zone of the meniscus of the knee is a material that may provide a uniform structure different in nature to other materials decellularised for scaffolds. ${ }^{11}$ It is an avascular tissue, known as the white zone of the meniscus. The two menisci of a knee are interfaces between the bones of the joints. Each meniscus increases the joint's stability for articulation, distributes the axial load, absorbs shock and provides lubrication to the knee joint. ${ }^{12}$ Menisci are thick, crescent-shaped (or almost circular) structures composed primarily of water, collagen and glycosaminoglycans (GAGs). The collagen is mostly type I collagen. The predominance of type I collagen distinguishes the fibrocartilage of menisci from articular cartilage, where type II collagen dominates. ${ }^{13}$ Collagen is primarily responsible for the strength of menisci. 
The arrangement of collagen in the meniscus contributes to the mechanical properties. There are two structural regions in menisci. The outer casing of the meniscus is highly structured while the middle zone is more anisotropic. The outer part consists of circumferentially aligned bundles, anchored by a small number of perpendicular radial tie fibers. ${ }^{14,15}$ The collagen fibrils are more aligned in the circumferential fibers than in fibers of the meniscal surface. ${ }^{16}$ This fiber arrangement enables a vertical compressive load to be transferred into circumferential "hoop" stresses. ${ }^{17}$ Circumferential strains resulting from a compressive load have been measured ranging from $1.5 \%$ to $2.9 \%$ in different parts of the meniscus under a load of three times bodyweight and at varying loading speeds. ${ }^{18}$ In another in vitro study, a compressive strain of $3.4 \%$ was found in the medial-lateral direction of a human meniscus, with average tensile strains of $1.4 \%$ and $3.5 \%$ in the anterior-posterior and superior-inferior directions, respectively, with an applied load equivalent to bodyweight. ${ }^{19}$ The strain on the collagen fibrils constituting part of these tissues was not measured in that work, rather it was the whole tissue strain, which is typically not a one-toone relationship with fibril strain in tissues. ${ }^{20}$

Menisci exhibit rate dependant behavior under compression. At high loading frequencies $(0.1-1 \mathrm{~Hz})$ the menisci behave as a rubber-like, elastic material while at lower frequencies $(0.01-0.03 \mathrm{~Hz})$ significant viscous dissipation occurs. ${ }^{21}$ While water is essentially noncompressible, it may gradually be exuded, contributing to the viscous dissipation observed at lower loading frequencies. GAGs contribute to this dynamic behaviour, both by acting to retain water and by cross-linking collagen. ${ }^{21-24}$ At high loading rates, the elastic loading of the collagen fibers allows the meniscus to store energy, ${ }^{25}$ like a fibrilreinforced poroelastic material. The relative contribution of collagen fibril strain and fluid pressure to the mechanical properties of menisci depends, therefore, not only on strain rate but also strain magnitude. Compression experiments suggest that all three components are responsible for the mechanical response under low strain, while collagen fibers are primarily responsible for the mechanical response under high strain. ${ }^{26}$

The purpose of the work here is to directly measure the orientation and strain in individual collagen fibrils during unconfined compression of meniscal fibrocartilage by SAXS measurement of the D-spacing change and fibril orientation. While there are well-established models of the structure of these materials, including under compression, this study enables for the first time the direct experimental monitoring and quantification of the strains imposed on collagen during compression in these tissues as a possible candidate for a source for a scaffold material. The meniscus material is not decellularised for this work, and no tests are made on decellularised material.

\section{Materials and Methods \\ Source Materials}

Bovine lateral meniscal fibrocartilage was sourced from the joints of the back limbs of two animals under 30 months castrated males from a licensed abattoir. The joint was dissected and the lateral meniscal fibrocartilage was removed by cutting the cranial ligament of the lateral meniscus, the meniscofemoral ligament, the cranial cruciate ligament and the underlying tissue.

Two samples, from both the lateral and medial meniscus in the joint, were cut from the middle zone in the positions indicated in Figure 1. Each sample was initially the full thickness of the lateral meniscus and then the top and bottom were sliced off, leaving a block with the dimensions as measured with a caliper as shown in Table 1 containing material from just the middle zone. The samples were stored in sealed bags for up to an hour before analysis.

\section{SAXS}

Diffraction patterns were recorded on the SAXS/WAXS beamline at the Australian Synchrotron, operated by ANSTO. This beamline utilizes a high-intensity undulator source and has an energy resolution of $10^{-4}$ from a cryocooled Si (111) double-crystal monochromator. The beam size (FWHM focused at the sample) was $250 \times 25 \mu \mathrm{m}$ with a total photon flux of $2 \times 10^{12} \mathrm{ph} \cdot \mathrm{s}^{-1}$. Using a Pilatus $2 \mathrm{M}$ detector with an active area of $254 \times 289 \mathrm{~mm}$, gapless diffraction patterns were recorded with an X-ray energy of $11.2 \mathrm{keV}$ and a sample to detector distance of $3384.9 \mathrm{~mm}$. Exposure time for diffraction patterns was $1 \mathrm{~s}$. Data were processed using the scatterBrain v2.82 Analysis software.

\section{SAXS Compression}

For the in-situ SAXS compression measurements, a tensile rig with stretching capabilities ${ }^{27}$ was modified, although the purpose-built frame and linear motor were left unaltered. The 3-kg capacity L6D aluminium alloy OIML single-point loadcell was replaced with a CAS $30-\mathrm{kg}$ capacity BCL-30L single point anodized aluminum loadcell. The two clamps used for stretching materials were replaced with custom-built plate attachments capable of 


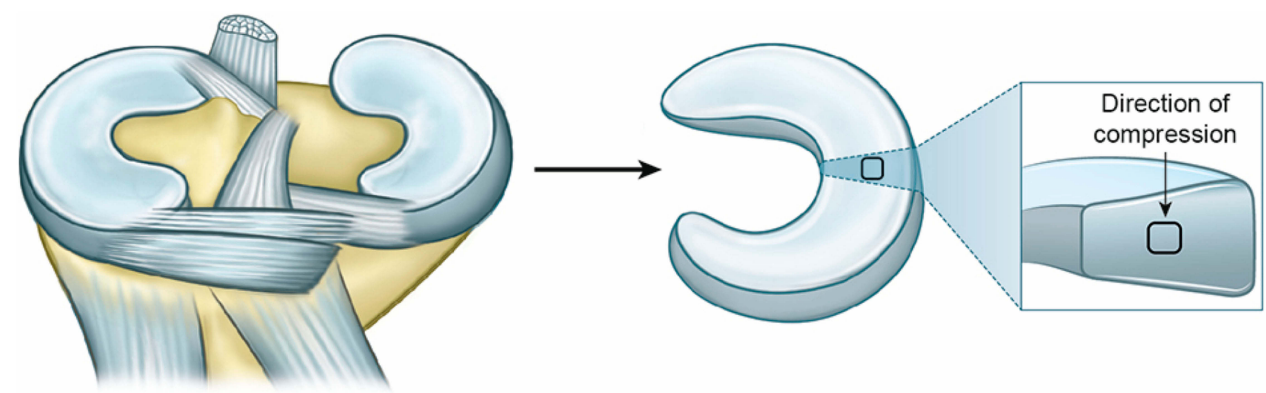

Figure I Lateral meniscus sampling positions.

compressing materials. The final device was mounted vertically in the beamline (Figure 2). Strain was recorded by the calibrated movement of the linear actuator divided by the initial sample thickness. The deflection of the load cell under load was small enough to be ignored.

Each meniscus sample was mounted on the bottom plate and then moved into the path of the X-ray beam. The top plate was lowered until it was just touching the top surface of the meniscus. Diffraction patterns were recorded in a horizontal line across the meniscus with steps of $1 \mathrm{~mm}$. Force and compression data were logged simultaneously. The top plate was then lowered by $0.5 \mathrm{~mm}$, the sample hydrated by an automated syringe with each compression increment, and force and compression information were again logged and SAXS spectra were recorded. This entire process (lower top plate $0.5 \mathrm{~mm}$, hydrate sample, record data and spectra) was repeated with a further $0.5 \mathrm{~mm}$ compression each time until the meniscus sample was compressed to about $1 \mathrm{~mm}$ (crushing the tissue past what would occur in-vivo). This limit was determined by the ability to get a clean X-ray diffraction pattern from the sample without interference from the compression jaws. The loading rate was $0.44-0.58 \mathrm{~mm} / \mathrm{min}$ and the unloading rate was $0.33-0.50 \mathrm{~mm} / \mathrm{min}$ or typically a load-unload frequency of $0.0014 \mathrm{~Hz}$, which is well into the viscous dissipation regime.

SAXS has been shown to be a useful method to measure stress in macromolecules ${ }^{28-30}$ including collagen which has the specific advantages of well-defined diffraction peaks. ${ }^{27,31}$

\section{D-Spacing}

The D-spacing was determined from Bragg's Law by taking the center of a Gaussian curve fitted to the 5th order diffraction peak of an integrated intensity plot for each pattern.

\section{Orientation Index}

Orientation Index (OI) is defined as $\left(90^{\circ}-\mathrm{OA}\right) / 90^{\circ}$, where $\mathrm{OA}$ is the azimuthal angle range that contains $50 \%$ of the fibrils centered around $180^{\circ}$. The OI quantifies the spread in orientation of the fibrils. An OI of 1 indicates the fibrils are completely parallel to one another while an OI of 0 indicates the fibrils are completely isotropically oriented. The OI was calculated around the 6th peak at $0.059-0.060 \AA^{-1} .^{32}$

\section{Results}

The stress-strain curves for the compression and release show a consistent elastic modulus over the range of strain experienced in this test (Figure 3). During compression, the stress strain curves are almost linear, then on release of the pressure, the response is non-linear with an initial rapid drop in stress. The material returns only partially toward the original thickness in these experiments. This work was done at what could be considered extremely low frequency, in the range where viscous dissipation is very important. $^{21,33}$ The maximum strain these tissues were subjected to of between 0.45 and 0.70 exceeds the strain experienced under physiological conditions (normally up

Table I Sample Measurements

\begin{tabular}{|l|l|l|l|l|l|}
\hline Sample Name & Leg (Left or Right) & Meniscus (Lateral or Medial) & Height (mm) & Width (mm) & Thickness (mm) \\
\hline A & Left & lateral & 5.90 & 5.19 & 7.30 \\
B & medial & 5.65 & 6.40 & 6.03 \\
C & meft & medial & 5.53 & 5.56 & 5.89 \\
D & Left & medial & 6.40 & 7.80 & 8.20 \\
E & Left & lateral & 4.85 & 7.97 & 5.81 \\
\hline
\end{tabular}



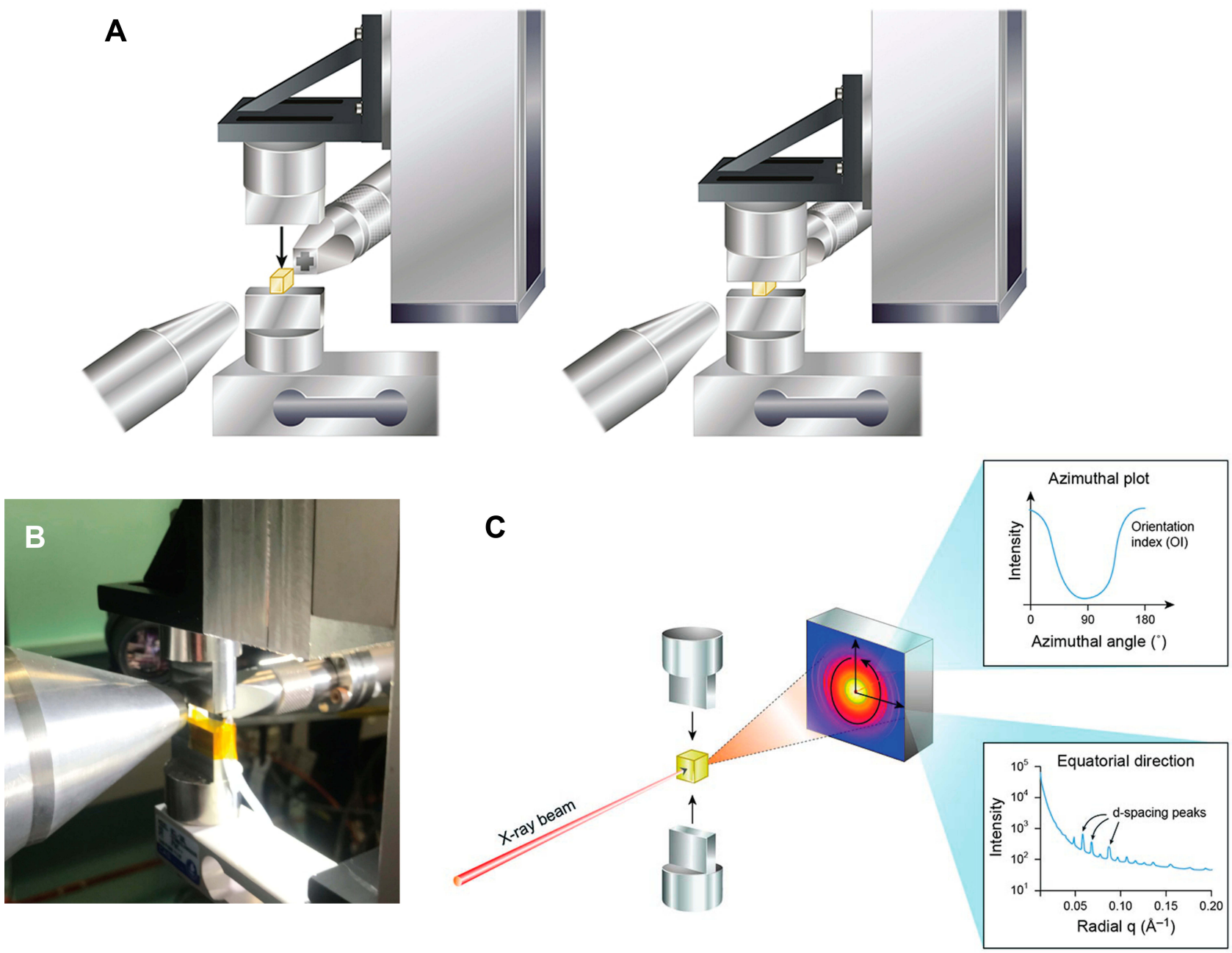

Figure 2 (A) Compression of meniscus on a load cell in the X-ray beam; (B) photo of sample being compressed during X-ray data collection; (C) experimental arrangement for data collection with azimuthal and equatorially integrated plots.

to 0.20 ), and therefore the relaxation curves may have limited physiological relevance, but are shown here for completeness.

The scattering patterns obtained during compression show distinct diffraction rings from the collagen D-banding (Figure 4). These rings are not of equal intensity at all azimuthal angles, which indicates the preferred orientation of the collagen fibrils (Figure 4A and B). Specifically, as the meniscus is compressed, the azimuthal angle subtended by these diffraction rings decreases (i.e. from Figure $4 \mathrm{~A}$ and $\mathrm{B}$ ) owing to the increasingly oriented collagen fibrils. Also with increasing compression, the diffraction peaks from the D-spacing of the meniscal collagen fibrils shifts (Figure 4C).

According to the SAXS results (Figure 5), there is good consistency in the shape of the OI and d-spacing curves with stress or strain with the five samples. Under compression, OI increases as the collagen fibrils reorient
(Figure 5A and C) and D-spacing increases as the collagen fibrils increase in length (Figure 5B and D). During compression the OI reaches a very high value $(0.7-0.85)$, reflecting very highly oriented fibrils (arranged as layers in the plane of the tissue). In all but one of the samples, this increase proceeds continuously with continuing compression (strain), only leveling off at high strain $(>0.4-0.5)$. The D-spacing also continues to increase as the compression force is increased and like the OI levels off at the highest strain. The fibrils therefore are extending in length as the meniscus is compressed.

These samples of meniscus were compressed while wet so that water was exuded from the tissue. On release of compression, the samples were maintained in an environment with free water on their surfaces so that there was an opportunity for reabsorption of water, as would be expected in a living biological system; however, the 


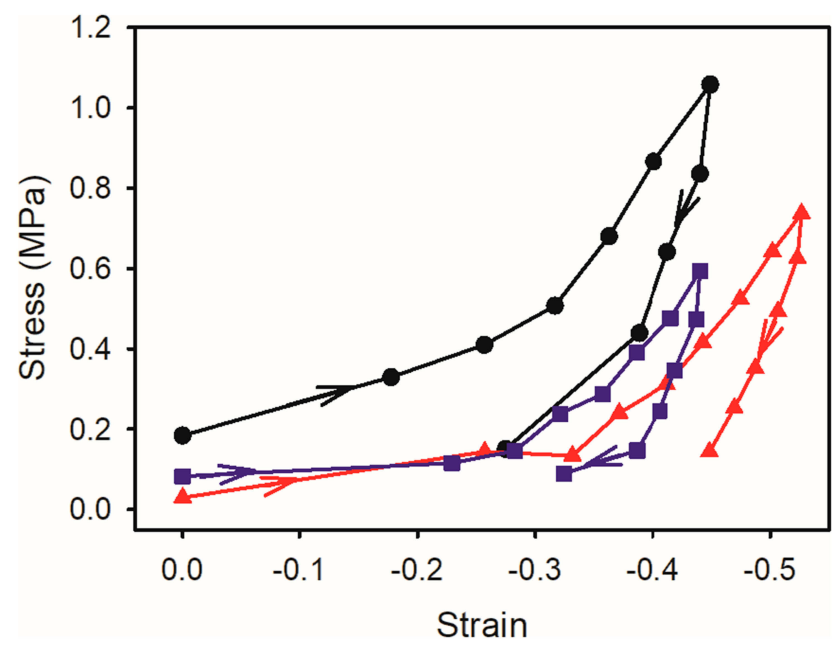

Figure 3 Stress-strain for meniscus compression and decompression for five samples (A black circles, B blue squares, C red up triangles, D dark green down triangles, $E$ dark-red diamonds). Arrows indicate the direction of time with the part of the line on the lower right representing the decompression stage.

meniscus samples did not elicit a perfectly elastic response (Figure 3).

During decompression, both the original OI and the original D-spacing were largely recovered.

\section{Discussion}

That meniscal fibrocartilage can transfer vertical compressive loads into tension in the circumferential direction has been observed experimentally ${ }^{17}$ and modelled. ${ }^{34-36}$ However, much of this load in the meniscus is taken up by the outer sheath of the meniscus, ${ }^{37}$ while the middle zone of the meniscus provides some resistance to compression to complement this. The more uniform nature of the middle zone material may make it suitable for the preparation of a scaffold material. ${ }^{11}$ Here, we have directly shown the extent of collagen fibril extension and rearrangement in the meniscus middle zone material during this compression using SAXS measurements of collagen fibrils that are a component of meniscus.

Under compression, the extension of the collagen fibrils was apparent as an increase in D-spacing. The fibril length increases by up to $2.3 \%$ (Figure $5 \mathrm{~B}$ ) for the maximum compression applied here. The compression strain experienced by the tissue was much greater than that typically experienced in the physiological range (up to 0.2 strain) likely largely due to the unconfined nature of the experiment.

Extension of collagen fibrils has similarly been observed in skin, pericardium and ovine forestomach that is stretched, ${ }^{27,31,38}$ with the difference that the strain is perpendicular to compression in meniscal fibrocartilage and parallel to tension in skin, pericardium and forestomach. The extension we observed in meniscus is less than the maximum reported for tissues under tensile strain (e.g. $6.7 \%$ in pericardium ${ }^{20}$ ) but is still a substantial increase. In skin and pericardium, the increase in D-spacing of the collagen fibrils is less than that of the whole tissue. ${ }^{27,31,38}$ This difference between fibril strain and tissue strain results from straightening of the collagen fibrils, a rearrangement of the fibrils and the sliding of the fibrils or fibers, which each add extension to the tissue but not the fibrils. Similarly, the collagen fibrils in menisci exhibit sliding, straightening or rearrangement when the tissue is strained as is also observed in skin and pericardium. Straightening and rearrangement are apparent as an increase in OI and may be accompanied by sliding of the collagen fibrils to enable this rearrangement. Both the collagen fibril rearrangement and the collagen fibril extension in compressed meniscus appear to be largely reversible, so that it is largely elastic under these experimental conditions, and this, therefore, provides a component of the elasticity of meniscal fibrocartilage under compression in biological systems (Figure 6).

In the decompression stage, the D-spacing largely follows the compression profile but decreases to a value slightly lower than the initial value. The unconfined meniscus experienced a strain of 0.8 in this work, much more than the strain of 0.2 maximally experienced in physiological conditions, so it is perhaps surprising that the recovery profile of the meniscus was as great as was observed. There is likely to be some irreversible damage to the structure of the tissue so that some pre-tension on the fibrils is released, however it may also be due to the water being forced out of the tissue and not being fully reabsorbed. It has been shown elsewhere that D-spacing is also dependent on hydration state. ${ }^{39,40}$ The differences observed between the different compression measurements experimentally measured here are likely due to the variation in the tissue samples rather than any factors associated with D-spacing measurement, OI measurement, strain rates or hydration.

The average pressure on meniscus in human knee joints under $100 \%$ bodyweight is approximately $1 \mathrm{MPa}$ (based on the estimated area of the meniscus of about $700 \mathrm{~mm}^{2}$ in $\mathrm{men}^{41}$ and a bodyweight of $700 \mathrm{~N}$ ). Previous studies have found the circumferential strains of the meniscus resulting from compressive loads of $1-3 x$ bodyweight (typical of those experienced by the joint) to be in the range $1.4 \%$ to $3.5 \%$ in different parts of the meniscus $^{18,19}$ (these bodyweights represent pressures of 

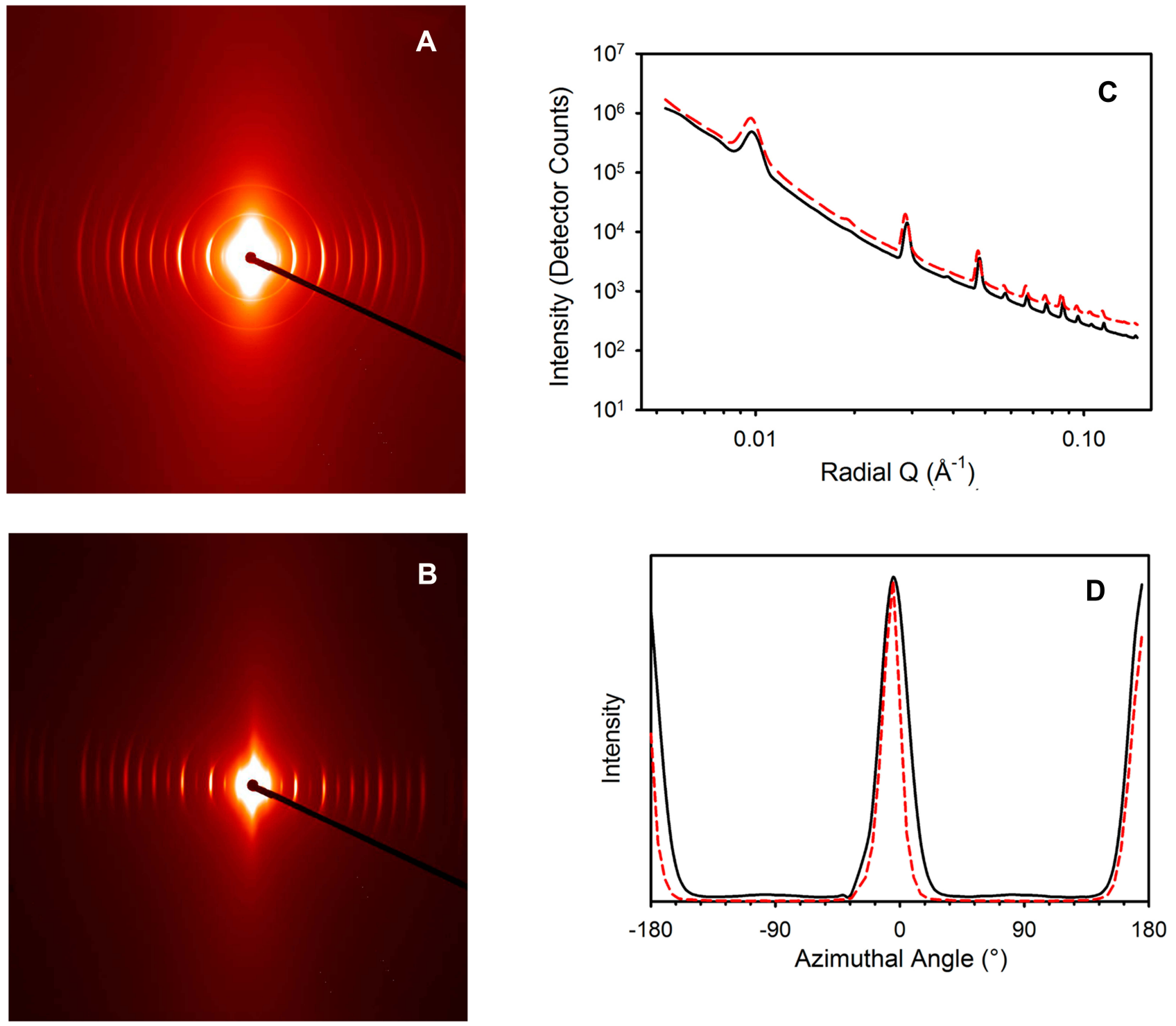

Figure 4 SAXS patterns for meniscus (A) before compression; (B) after compression; Integrated intensity; (C) for all radial angles (at q = $0.05 \AA^{-1}$ ); (D) for all azimuthal angles; before (black solid line) and after (red dashed line) compression (with (C) offset for clarity).

around 1-3 $\mathrm{MPa}$ ). The maximum pressures reached in the work presented here range up to $0.43 \mathrm{MPa}$. However in this work, the compression was unconfined, unlike under physiological conditions where the meniscus is confined, and at these pressures, the meniscus was strained up to 0.8 , much greater than is observed in confined meniscus.

The rate-dependent behavior of collagen under compression means that at slow loading rates, water is slowly exuded from meniscal tissue, mediated by GAGs, until, at high strain, the fibrils become responsible for the mechanical response. ${ }^{25,26}$ Some of this water may originate from within collagen fibrils given that fibril elasticity translates to fibril diameter changes, as determined elsewhere in other tissues using Poisson's ratio. ${ }^{31}$ The reported
Poisson's ratio of 2.1 for collagen in pericardium under tension is higher than that possible for isotropic closed systems, so it is possible that the movement of water into and out of the fibrils is responsible for this Poisson's ratio. A Poisson's ratio of greater than 0.5 for collagen fibril extension means that fibril volume decreases as they undergo tension (and hence water is exuded from the fibril as the diameter decreases and the volume decreases), whereas for a closed system (e.g. a rod of isotropic material) the volume must increase as tension is applied. A volume decrease under tension might be possible for a non-isotropic structure that collapses on itself under tension (in the case of collagen, tightening of the coiled structure) and loses water. Specifically, the counter-twisted 

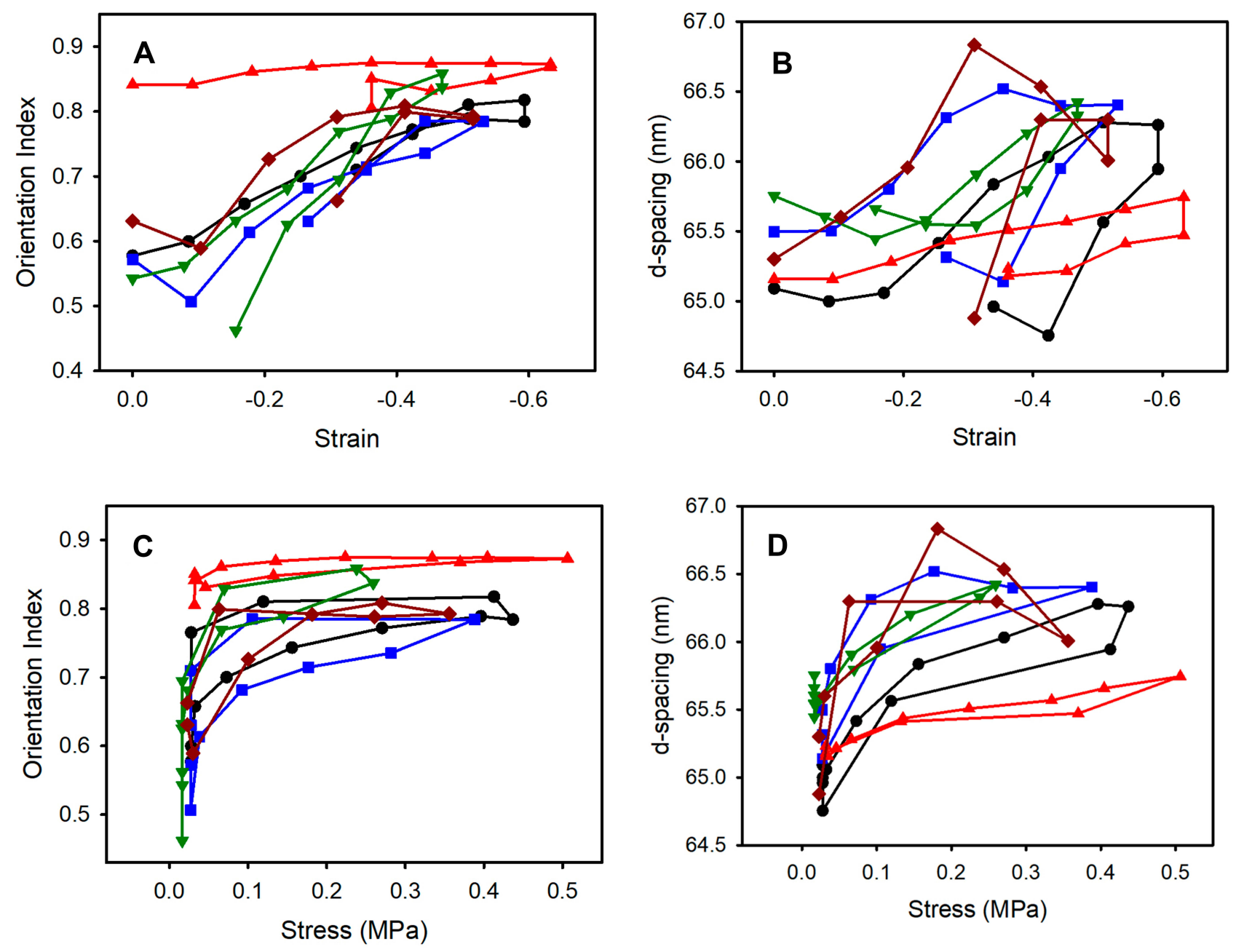

Figure 5 Change in collagen fibril structure under increasing compression (and decompression) for five samples (A black circles, B blue squares, C red up triangles, D dark green down triangles, E dark-red diamonds): (A) Ol vs compressive linear strain; (B) D-spacing vs compressive linear strain; (C) Ol vs stress; (D) D-spacing vs stress. The upper part of each line is the compression phase while the lower part is the decompression stage.

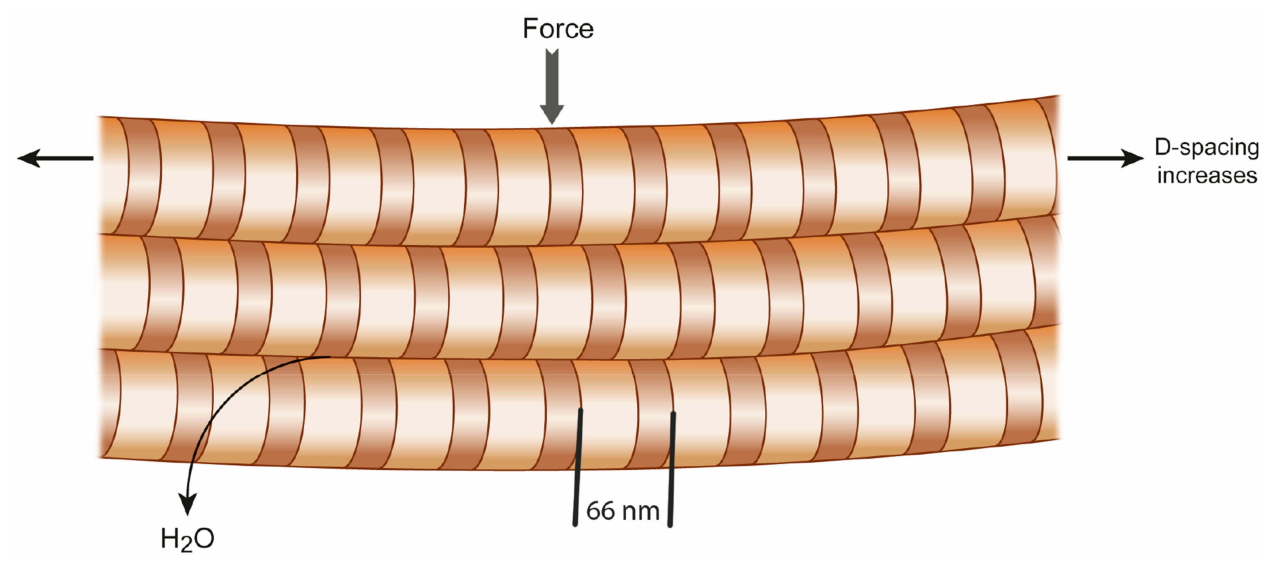

Figure 6 Graphical illustration of the elasticity of meniscal fibrocartilage collagen under compression.

nature of tropocollagen (left-handed coil for the collagen molecule and right-handed turn of the three collagen molecules that make up tropocollagen) would assist in directing the applied tensile forces into a fibril diameter contraction, in a similar manner to a hawser-laid rope. The loss of water from collagen may lead to or be caused by 
a reduction in fibril diameter with a possible source of that water being the hydrogen bond network within the fibrils, so that compression of this structure may release this water. However, water has also been seen to affect the D-spacing of collagen such that a reduction in water content results in a reduction in the D-spacing, ${ }^{39,42}$ although the D-spacing is also affected by the tension applied to the fibrils.

The observed increase in fiber alignment in planes perpendicular to the direction of force, as indicated by increasing OI, is similar to that seen in acellular dermal matrix or leather when stretched, ${ }^{27,31,38}$ with the contrast that the alignment becomes perpendicular to compression in meniscus and parallel to tension in skin. However, both result in a more oriented collagen material where the fibrils have rearranged in the direction of the positive strain. Compression of skin has also been shown to lead to an increase in OI in one study, but the differences in orientation were rather small. ${ }^{43}$ This could in part be attributed to simple geometrical consideration with the change in thickness rather than any rearrangement (in other words to affine transformation). ${ }^{4,45}$

Compression of the meniscal tissue in a confined space translates to tensile stress at right angles to the direction of this force. After some initial straightening and orienting in the direction perpendicular to the direction of compression, the fibrils take up this stress by extending along their length, accompanied by some loss of water (Figure 6), with the water loss mediated by GAG content. ${ }^{24}$ The degree of elasticity of the collagen fibrils in tension along their length when the meniscus is compressed, therefore, contributes to the elastic response of the meniscus. This necessitates the fibril arrangement seen in nature - they are perpendicular to the direction of compression. The shape of the collagen fibrils, long thin rods, is used to maximum advantage. Consider an alternative where the fibrils were to be arranged where they would be oriented in the direction of the compressive force - this would be ineffective at resisting the force because the flexible fibrils would buckle and bend, and provide very little resistance to the force. However, with the fibril aligned at right angles to the compressive force, they can transmit this force to a positive strain in the fibrils. Collagen is well suited to responding elastically, with a high elastic modulus and high tensile strength. This is just one part of the compression resistance of meniscus. Here we have shown the extent of strain in individual collagen fibrils during compression, and measured the rearrangement of collagen fibrils.
If this tissue is to be used for a scaffold material, then a process of decellularisation is necessary. This will involve the removal of all cellular material but at the same time other components such as GAGs. These are likely to have some effect on the mechanical properties of the material. In addition, there may be some changes to the collagen matrix. Then, the important consideration of how well these scaffolds integrate into the body must be addressed. Such issues have been encountered in other decellularised collagen materials. ${ }^{2}$ Here we have provided the first step in this journey, by characterizing the native material under compression.

\section{Conclusions}

The structure of the middle zone of bovine meniscus and the behavior of collagen fibrils in this material with unconfined compression were investigated. The collagen fibrils in the middle zone became more highly oriented perpendicular to the direction of compression. The D-spacing increased as a result of the elongation of collagen fibrils perpendicular to compression. The elasticity of the collagen fibrils under tension along their length when the meniscus is compressed, therefore, contributes to the overall elastic response of the meniscus but only under loads that exceed those likely to be experienced physiologically. This material may be suitable, after decellularisation, as a surgical scaffold material in applications that, for example, requires a tissue suitable to cope with compressive forces.

\section{Acknowledgments}

This research was undertaken on the SAXS/WAXS beamline at the Australian Synchrotron, part of ANSTO. The New Zealand Synchrotron Group provided travel and accommodation.

\section{Disclosure}

Richard G Haverkamp reports grants from Australian Synchrotron, during the conduct of the study. The authors report no other possible conflicts of interest in this work.

\section{References}

1. Murphy CA, Garg AK, Silva-Correia J, Reis RL, Oliveira JM, Collins MN. The meniscus in normal and osteoarthritic tissues: facing the structure property challenges and current treatment trends. Ann Rev Biomed Eng. 2019;21(1):495-521. doi:10.1146/annurev-bioeng $-060418-052547$

2. Murphy CA, Costa JB, Silva-Correia J, Oliveira JM, Reis RL, Collins MN. Biopolymers and polymers in the search of alternative treatments for meniscal regeneration: state of the art and future trends. Appl Mater Today. 2018;12:51-71. doi:10.1016/j.apmt.2018.04.002 
3. Keane TJ, Badylak SF. Biomaterials for tissue engineering applications. Semin Pediatr Surg. 2014;23(3):112-118. doi:10.1053/ j.sempedsurg.2014.06.010

4. O'Brien FJ. Biomaterials \& scaffolds for tissue engineering. Mater Today. 2011;14(3):88-95. doi:10.1016/S1369-7021(11)70058-X

5. Sachlos E, Czernuszka JT, Gogolewski S, Dalby M. Making tissue engineering scaffolds work. Review on the application of solid freeform fabrication technology to the production of tissue engineering scaffolds. Eur Cells Mater. 2003;5:29-40. doi:10.22203/eCM.v005a03

6. Wang Y, Blasioli DJ, Kim HJ, Kim HS, Kaplan DL. Cartilage tissue engineering with silk scaffolds and human articular chondrocytes. Biomaterials. 2006;27(25):4434 4442. doi:10.1016/j.biomaterials.2006.03.050

7. Faulk DM, Wildemann JD, Badylak SF. Decellularization and cell seeding of whole liver biologic scaffolds composed of extracellular matrix. J Clin Exp Hepatol. 2015;5(1):69-80. doi:10.1016/j. jceh.2014.03.043

8. Weng T, Wu P, Zhang W, et al. Regeneration of skin appendages and nerves: current status and further challenges. J Transl Med. 2020;18 (1). doi:10.1186/s12967-020-02248-5.

9. Wells HC, Sizeland KH, Kirby N, Hawley A, Mudie S, Haverkamp RG. Collagen fibril structure and strength in acellular dermal matrix materials of bovine, porcine and human origin. ACS Biomat Sci Eng. 2015;1(10):1026-1038. doi:10.1021/acsbiomateri als.5b00310

10. Floden EW, Malak S, Basil-Jones MM, et al. Biophysical characterization of ovine forestomach extracellular matrix biomaterials. J Biomed Mat Res B. 2010;96B(1):67-75. doi:10.1002/jbm.b.31740

11. Yusof F, Sha'ban M, Azhim A. Development of decellularized meniscus using closed sonication treatment system: potential scaffolds for orthopedics tissue engineering applications. Int $J$ Nanomed. 2019;14:5491-5502. doi:10.2147/IJN.S207270

12. Fox AJS, Wanivenhaus F, Burge AJ, Warren RF, Rodeo SA. The human meniscus: a review of anatomy, function, injury, and advances in treatment. Clin Anat. 2015;28(2):269-287. doi:10.1002/ca.22456

13. Fox AJS, Bedi A, Rodeo SA. The basic science of human knee menisci: structure, composition, and function. Sports Health. 2012;4 (4):340-351. doi:10.1177/1941738111429419

14. Aspden RM, Yarker YE, Hukins DWL. Collagen orientations in the meniscus of the knee joint. J Anat. 1985;140(3):371-380.

15. Kambic HE, McDevitt CA. Spatial organization of types I and II collagen in the canine meniscus. J Orthop Res. 2005;23(1):142-149. doi:10.1016/j.orthres.2004.06.016

16. Li Q, Qu F, Han B, et al. Micromechanical anisotropy and heterogeneity of the meniscus extracellular matrix. Acta Biomater. 2017;54:356-366. doi:10.1016/j.actbio.2017.02.043

17. Ghosh P, Ingman AM, Taylor TK. Variations in collagen, non-collagenous proteins, and hexosamine in menisci derived from osteoarthritic and rheumatoid arthritic knee joints. J Rheumatol. 1975;2(1):100-107.

18. Spencer Jones R, Keene GCR, Learmonth DJA, et al. Direct measurement of hoop strains in the intact and torn human medial meniscus. Clin Biomech. 1996;11(5):295-300. doi:10.1016/0268-0033(96)00003-4

19. Kolaczek S, Hewison C, Caterine S, Ragbar MX, Getgood A, Gordon KD. Analysis of 3D strain in the human medial meniscus. J Mech Behav Biomed. 2016;63:470-475. doi:10.1016/j.jmbbm.2016.06.001

20. Kayed HR, Kirby N, Hawley A, Mudie ST, Haverkamp RG. Collagen fibril strain, recruitment and orientation for pericardium under tension and the effect of cross links. RSC $A d v$. 2015;5:103703-103712. doi:10.1039/C5RA21870E

21. Bursac P, Arnoczky S, York A. Dynamic compressive behavior of human meniscus correlates with its extra-cellular matrix composition. Biorheology. 2009;46(3):227-237. doi:10.3233/BIR-2009-0537

22. Haverkamp RG, Marshall AT, Williams MAK. Model for stretching elastic biopolymers which exhibit conformational transformations. Phys Rev E. 2007;75:021907/021901-021907/021907. doi:10.1103/ PhysRevE.75.021907
23. Haverkamp RG, Williams MAK, Scott JE. Stretching single molecules of connective tissue glycans to characterize their shape-maintaining elasticity. Biomacromolecules. 2005;6 (3):1816-1818. doi:10.1021/bm0500392

24. Murphy CA, Cunniffe GM, Garg AK, Collins MN. Regional dependency of bovine meniscus biomechanics on the internal structure and glycosaminoglycan content. J Mech Behav Biomed. 2019;94:186-192. doi:10.1016/j.jmbbm.2019.02.020

25. Danso EK, Mäkelä JTA, Tanska P, et al. Characterization of site-specific biomechanical properties of human meniscus-Importance of collagen and fluid on mechanical nonlinearities. $J$ Biomech. 2015;48 (8):1499-1507. doi:10.1016/j.jbiomech.2015.01.048

26. Gabrion A, Aimedieu P, Laya Z, et al. Relationship between ultrastructure and biomechanical properties of the knee meniscus. Surg Radiol Anat. 2005;27(6):507-510. doi:10.1007/s00276-005-0031-6

27. Basil-Jones MM, Edmonds RL, Norris GE, Haverkamp RG. Collagen fibril alignment and deformation during tensile strain of leather: A SAXS study. J Agric Food Chem. 2012;60(5):1201-1208. doi:10.1021/jf2039586

28. Collins MN, Dalton E, Schaller B, Leahy JJ, Birkinshaw C. Crystal morphology of strained ultra high molecular weight polyethylenes. Polym Test. 2012;31(5):629-637. doi:10.1016/j.polymertesting.2012.03.009

29. Collins MN, Dalton E, Leahy JJ, Birkinshaw C. Effects of tensile strain on the nanostructure of irradiated and thermally stabilised ultra high molecular weight polyethylenes for orthopaedic devices. RSC Adv. 2013;3(6):1995-2007. doi:10.1039/C2RA21722H

30. Dalton E, Collins MN. Lamella alignment ratio: A SAXS analysis technique for macromolecules. J Appl Crystallogr. 2014;47 (3):847-851. doi:10.1107/S1600576714005706

31. Wells HC, Sizeland KH, Kayed HR, et al. Poisson's ratio of collagen fibrils measured by SAXS of strained bovine pericardium. $J$ Appl Phys. 2015;117(4):044701. doi:10.1063/1.4906325

32. Basil-Jones MM, Edmonds RL, Allsop TF, et al. Leather structure determination by small angle X-ray scattering (SAXS): cross sections of ovine and bovine leather. J Agric Food Chem. 2010;58 (9):5286-5291. doi:10.1021/jf100436c

33. Rodriguez ML, Li L. Compression-rate-dependent nonlinear mechanics of normal and impaired porcine knee joints. $B M C$ Musculoskelet Disord. 2017;18(1). doi:10.1186/s12891-017-1805-9

34. Orozco GA, Tanska P, Mononen ME, Halonen KS, Korhonen RK The effect of constitutive representations and structural constituents of ligaments on knee joint mechanics. Sci Rep-UK. 2018;8 (1):1-5.

35. Freutel M, Galbusera F, Ignatius A, Dürselen L. Material properties of individual menisci and their attachments obtained through inverse FE-analysis. $J$ Biomech. 2015;48(8):1343-1349. doi:10.1016/j. jbiomech.2015.03.014

36. Seitz AM, Galbusera F, Krais C, Ignatius A, Dürselen L. Stressrelaxation response of human menisci under confined compression conditions. J Mech Behav Biomed. 2013;26:68-80. doi:10.1016/j. jmbbm.2013.05.027

37. Abraham AC, Edwards CR, Odegard GM, Donahue TLH. Regional and fiber orientation dependent shear properties and anisotropy of bovine meniscus. J Mech Behav Biomed. 2011;4(8):2024-2030. doi:10.1016/j.jmbbm.2011.06.022

38. Sizeland KH, Wells HC, Kelly SJR, et al. The collagen fibril response to strain in scaffolds from ovine forestomach for tissue engineering. ACS Biomat Sci Eng. 2017;3(10):2550-2558. doi:10.1021/ acsbiomaterials. $7 \mathrm{~b} 00588$

39. Sizeland KH, Edmonds RL, Wells HC, et al. The influence of water, lanolin, urea, proline, paraffin and fatliquor on collagen D-spacing in leather. $R S C A d v$. 2017;7(64):40658-40663. doi:10.1039/C7RA05560A

40. Kelly SJR, Weinkamer R, Bertinetti L, et al. Effect of collagen packing and moisture content on leather stiffness. J Mech Behav Biomed. 2019;90:1-10. doi:10.1016/j.jmbbm.2018.10.004 
41. Bloecker K, Englund M, Wirth W, et al. Revision 1 Size and position of the healthy meniscus, and its Correlation with sex, height, weight, and bone area- a cross-sectional study. BMC Musculoskelet Disord. 2011;12:248.

42. Sizeland KH, Holmes G, Edmonds RL, et al. Fatliquor effects on collagen fibril orientation and d-spacing in leather during tensile strain. J Am Leather Chem Assoc. 2015;110(11):355-362.

43. Hepworth DG, Steven-fountain A, Bruce DM, Vincent JFV. Affine versus non-affine deformation in soft biological tissues, measured by the reorientation and stretching of collagen fibres through the thickness of compressed porcine skin. J Biomech. 2001;34(3):341-346. doi:10.1016/S0021-9290(00)00183-4
44. Sizeland KH, Edmonds RL, Basil -jones MM, et al. Changes to collagen structure during leather processing. J Agric Food Chem. 2015;63(9):2499-2505. doi:10.1021/jf506357j

45. Kelly SJ, Wells HC, Sizeland KH, et al. Artificially modified collagen fibril orientation affects leather tear strength. J Sci Food Agric. 2018;98(9):3524-3531. doi:10.1002/jsfa.8863

\section{Publish your work in this journal}

The International Journal of Nanomedicine is an international, peerreviewed journal focusing on the application of nanotechnology in diagnostics, therapeutics, and drug delivery systems throughout the biomedical field. This journal is indexed on PubMed Central, MedLine, CAS, SciSearch ${ }^{\mathbb{R}}$, Current Contents ${ }^{\mathbb{R}} /$ Clinical Medicine, $^{-}$
Journal Citation Reports/Science Edition, EMBase, Scopus and the Elsevier Bibliographic databases. The manuscript management system is completely online and includes a very quick and fair peer-review system, which is all easy to use. Visit http://www.dovepress.com/ testimonials.php to read real quotes from published authors. 